\title{
Discrepancy of Symmetric Products of Hypergraphs
}

\author{
Benjamin Doerr* $\quad$ Michael Gnewuch ${ }^{\dagger} \quad$ Nils Hebbinghaus ${ }^{\ddagger}$
}

Submitted: Dec 15, 2005; Accepted: Mar 28, 2006; Published: Apr 24, 2006

Mathematics Subject Classification: 11K38

\begin{abstract}
For a hypergraph $\mathcal{H}=(V, \mathcal{E})$, its $d$-fold symmetric product is defined to be $\Delta^{d} \mathcal{H}=\left(V^{d},\left\{E^{d} \mid E \in \mathcal{E}\right\}\right)$. We give several upper and lower bounds for the $c$-color discrepancy of such products. In particular, we show that the bound $\operatorname{disc}\left(\Delta^{d} \mathcal{H}, 2\right) \leq$ $\operatorname{disc}(\mathcal{H}, 2)$ proven for all $d$ in [B. Doerr, A. Srivastav, and P. Wehr, Discrepancy of Cartesian products of arithmetic progressions, Electron. J. Combin. 11(2004), Research Paper 5, 16 pp.] cannot be extended to more than $c=2$ colors. In fact, for any $c$ and $d$ such that $c$ does not divide $d$ !, there are hypergraphs having arbitrary large discrepancy and $\operatorname{disc}\left(\Delta^{d} \mathcal{H}, c\right)=\Omega_{d}\left(\operatorname{disc}(\mathcal{H}, c)^{d}\right)$. Apart from constant factors (depending on $c$ and $d$ ), in these cases the symmetric product behaves no better than the general direct product $\mathcal{H}^{d}$, which satisfies $\operatorname{disc}\left(\mathcal{H}^{d}, c\right)=O_{c, d}\left(\operatorname{disc}(\mathcal{H}, c)^{d}\right)$.
\end{abstract}

\section{Introduction}

We investigate the discrepancy of certain products of hypergraphs. In [3], Srivastav, Wehr and the first author noted the following. For a hypergraph $\mathcal{H}=(V, \mathcal{E})$ define the $d$-fold direct product and the $d$-fold symmetric product by

$$
\begin{aligned}
\mathcal{H}^{d} & :=\left(V^{d},\left\{E_{1} \times \cdots \times E_{d} \mid E_{i} \in \mathcal{E}\right\}\right), \\
\Delta^{d} \mathcal{H} & :=\left(V^{d},\left\{E^{d} \mid E \in \mathcal{E}\right\}\right) .
\end{aligned}
$$

*Max-Planck-Institut für Informatik, Saarbrücken, Germany.

$\dagger$ Institut für Informatik und Praktische Mathematik, Christian-Albrechts-Universität zu Kiel, Germany. Supported by the Deutsche Forschungsgemeinschaft, Grant SR7/10-1.

$\ddagger$ Max-Planck-Institut für Informatik, Stuhlsatzenhausweg 85, 66123 Saarbrücken, Germany, Tel.: +49-681/9325-109, E-mail: nhebbing@mpi-inf.mpg.de. (corresponding author) 
Then for the (two-color) discrepancy

$$
\operatorname{disc}(\mathcal{H}):=\min _{\chi: V \rightarrow\{-1,1\}} \max _{E \in \mathcal{E}}\left|\sum_{v \in E} \chi(v)\right|,
$$

we have

$$
\begin{aligned}
\operatorname{disc}\left(\mathcal{H}^{d}\right) & \leq \operatorname{disc}(\mathcal{H})^{d} \\
\operatorname{disc}\left(\Delta^{d} \mathcal{H}\right) & \leq \operatorname{disc}(\mathcal{H}) .
\end{aligned}
$$

In this paper, we show that the situation is more complicated for discrepancies in more than two colors. In particular, it depends highly on the dimension $d$ and the number of colors, whether the discrepancy of symmetric products is more like the discrepancy of the original hypergraph or the $d$-th power thereof. Let us make this precise:

Let $\mathcal{H}=(V, \mathcal{E})$ be a hypergraph, that is, $V$ is some finite set and $\mathcal{E} \subseteq 2^{V}$. Without loss of generality, we will assume that $V=[n]$ for some $n \in \mathbb{N}$. Here and in the following we use the shorthand $[r]:=\{n \in \mathbb{N} \mid n \leq r\}$ for any $r \in \mathbb{R}$. The elements of $V$ are called vertices, those of $\mathcal{E}$ (hyper)edges. For $c \in \mathbb{N}_{\geq 2}$, a $c$-coloring of $\mathcal{H}$ is a mapping $\chi: V \rightarrow[c]$. The discrepancy problem asks for balanced colorings of hypergraphs in the sense that each hyperedge shall contain the same number of vertices in each color. The discrepancy of $\chi$ and the $c$-color discrepancy of $\mathcal{H}$ are defined by

$$
\begin{aligned}
\operatorname{disc}(\mathcal{H}, \chi) & :=\max _{E \in \mathcal{E}} \max _{i \in[c]}|| \chi^{-1}(i) \cap E\left|-\frac{1}{c}\right| E||, \\
\operatorname{disc}(\mathcal{H}, c) & :=\min _{\chi: V \rightarrow[c]} \operatorname{disc}(\mathcal{H}, \chi) .
\end{aligned}
$$

These notions were introduced in [2] extending the discrepancy problem for hypergraphs to arbitrary numbers of colors (see, e.g., the survey of Beck and Sós [1]). Note that $\operatorname{disc}(\mathcal{H})=2 \operatorname{disc}(\mathcal{H}, 2)$ holds for all $\mathcal{H}$. In this more general setting, the product bound proven in [3] is

$$
\operatorname{disc}\left(\mathcal{H}^{d}, c\right) \leq c^{d-1} \operatorname{disc}(\mathcal{H}, c)^{d}
$$

However, as we show in this paper the relation $\operatorname{disc}\left(\Delta^{d} \mathcal{H}, c\right)=O(\operatorname{disc}(\mathcal{H}, c))$ does not hold in general. In Section 2, we give a characterization of those values of $c$ and $d$, for which it is satisfied for every hypergraph $\mathcal{H}$. In particular, we present for all $c, d, k$ such that $c$ does not divide $d$ ! a hypergraph $\mathcal{H}$ having $\operatorname{disc}(\mathcal{H}, c) \geq k$ and $\operatorname{disc}\left(\Delta^{d} \mathcal{H}, c\right)=\Omega_{d}\left(k^{d}\right)$. In the light of (1), this is largest possible apart from factors depending on $c$ and $d$ only.

On the other hand, there are further situations where this worst case does not occur. We prove some in Section 3, but the complete picture seems to be complicated. 


\section{Coloring Simplices}

To get some intuition of what we do in the remainder, let us regard some small examples first. For $c=2$ colors and dimension $d=2$, it is easy to see that $\operatorname{disc}\left(\Delta^{d} \mathcal{H}, c\right) \leq \operatorname{disc}(\mathcal{H}, c)$ holds for arbitrary hypergraphs $\mathcal{H}=(V, \mathcal{E})$. As mentioned above, we assume for simplicity that $V=[n]$. Now coloring the vertices above the diagonal in one color, the ones below in the other, and those on the diagonal according to an optimal coloring for the onedimensional case does the job. More formally, let $\chi: V \rightarrow[2]$. Let $\tilde{\chi}: V^{2} \rightarrow[2]$ such that $\tilde{\chi}((x, y))=1$, if $x<y$, $\tilde{\chi}((x, y))=2$, if $x>y$, and $\tilde{\chi}((x, y))=\chi(x)$, if $x=y$. Then $\operatorname{disc}\left(\Delta^{2} \mathcal{H}, \tilde{\chi}\right)=\operatorname{disc}(\mathcal{H}, \chi)$. Hence $\operatorname{disc}\left(\Delta^{2} \mathcal{H}, 2\right) \leq \operatorname{disc}(\mathcal{H}, 2)$. This argument can be extended to arbitrary dimension to show $\operatorname{disc}\left(\Delta^{d} \mathcal{H}, 2\right) \leq \operatorname{disc}(\mathcal{H}, 2)$ for all $d \in \mathbb{N}$.

Things become more interesting if we do not restrict ourselves to 2 colors. For example, it is not clear how to extend the simple above/below diagonal approach to 3 colors (in two dimensions). In fact, as we will show in the following, such bounds do not exist for many pairs $(c, d)$, including $(3,2)$. However, in three dimensions $\operatorname{disc}\left(\Delta^{3} \mathcal{H}, 3\right) \leq \operatorname{disc}(\mathcal{H}, 3)$ follows similarly to the $(2,2)$ proof above. Indeed, for $c=2$ and $d=2$ we divided the product set $V^{2}$ into the sets above and below the diagonal, which we want to call twodimensional simplices of $V^{2}$, and the diagonal, a one-dimensional simplex of $V^{2}$. For $c=3$ and $d=3$ we divide $V^{3}$ into the six three-dimensional simplices in $V^{3}$ that we obtain from the set $\left\{x \in V^{3} \mid x_{1}<x_{2}<x_{3}\right\}$ by permuting coordinates, the six two-dimensional simplices in $V^{3}$ that we obtain from $\left\{x \in V^{3} \mid x_{1}=x_{2}<x_{3}\right\}$ by permuting coordinates and possibly changing $<$ to $>$, and finally the one-dimensional simplex $\left\{x \in V^{3} \mid x_{1}=\right.$ $\left.x_{2}=x_{3}\right\}$. Now with each color we color exactly two three-dimensional and two twodimensional simplices of $V^{3}$. The vertices of the diagonal will again be colored according to an optimal coloring for the one-dimensional case.

We shall now give a formal definition of $l$-dimensional simplices in arbitrary dimensions. A set $\left\{x_{1}, \ldots, x_{k}\right\}$ of integers with $x_{1}<\ldots<x_{k}$ is denoted by $\left\{x_{1}, \ldots, x_{k}\right\}<$. For a set $S$ we put

$$
\left(\begin{array}{l}
S \\
k
\end{array}\right)=\{T \subseteq S|| T \mid=k\}
$$

Furthermore, let $S_{k}$ be the symmetric group on $[k]$. For $l, d \in \mathbb{N}$ with $l \leq d$ let $P_{l}(d)$ be the set of all partitions of $[d]$ into $l$ non-empty subsets. Let $e_{1}=(1,0, \ldots, 0), \ldots$, $e_{d}=(0, \ldots, 0,1)$ be the standard basis of $\mathbb{R}^{d}$. For $c \in \mathbb{N}$ and $\lambda \in \mathbb{N}_{0}$ we write $c \mid \lambda$ if there exists an $m \in \mathbb{N}_{0}$ with $m c=\lambda$.

Definition 1. Let $d \in \mathbb{N}, l \in[d]$ and $T \subseteq \mathbb{N}$ finite. For $J=\left\{J_{1}, \ldots, J_{l}\right\} \in P_{l}(d)$ with $\min J_{1}<\ldots<\min J_{l}$ put $f_{i}=f_{i}(J)=\sum_{j \in J_{i}} e_{j}, i=1, \ldots, l$. Let $\sigma \in S_{l}$. We call

$$
S_{J}^{\sigma}(T):=\left\{\sum_{i=1}^{l} \alpha_{\sigma(i)} f_{i}(J) \mid\left\{\alpha_{1}, \ldots, \alpha_{l}\right\}<\subseteq T\right\}
$$


an $l$-dimensional simplex in $T^{d}$. If $l=d$, we simply write $S^{\sigma}(T)$ instead of $S_{J}^{\sigma}(T)$ (as $\left.\left|P_{d}(d)\right|=1\right)$.

Clearly, the simplices in a $d$-dimensional grid $T^{d}$ form a partition of $T^{d}$. The next remark shows that the numbers of $l$-dimensional simplices are well-understood.

Remark 2. If $S(d, l), d, l \in \mathbb{N}$, denote the Stirling numbers of the second kind, then $\left|P_{l}(d)\right|=S(d, l)$ (see, e.g. [6]). We have

$$
S(d, l)=\sum_{j=0}^{l} \frac{(-1)^{j}(l-j)^{d}}{j !(l-j) !} .
$$

Let $T \subseteq \mathbb{N}$ finite. Furthermore, let $I, J \in P_{l}(d)$ and $\sigma, \tau \in S_{l}$. If $|T| \geq l$, we have $S_{I}^{\sigma}(T) \neq S_{J}^{\tau}(T)$ as long as $I \neq J$ or $\sigma \neq \tau$. Thus the number of l-dimensional simplices in $T^{d}$ is $l ! S(d, l)$. If $|T|<l$, then there exists obviously no non-empty l-dimensional simplex in $T^{d}$.

We are now able to prove the main result of this paper.

Theorem 3. Let $c, d \in \mathbb{N}$.

(i) If $c \mid k ! S(d, k)$ for all $k \in\{2, \ldots, d\}$, then every hypergraph $\mathcal{H}$ satisfies

$$
\operatorname{disc}\left(\Delta^{d} \mathcal{H}, c\right) \leq \operatorname{disc}(\mathcal{H}, c) .
$$

(ii) If $c \nmid k ! S(d, k)$ for some $k \in\{2, \ldots, d\}$, then there exists a hypergraph $\mathcal{K}$ such that

$$
\operatorname{disc}\left(\Delta^{d} \mathcal{K}, c\right) \geq \frac{1}{3 k !} \operatorname{disc}(\mathcal{K}, c)^{k},
$$

and $\mathcal{K}$ can be chosen to have arbitrary large discrepancy $\operatorname{disc}(\mathcal{K}, c)$.

Before proving the theorem, we state some consequences. In particular, (3) holds never for $c=4$. For $c=3$, it holds exactly if $d$ is odd.

Corollary 4. (a) Let $d \geq 3$ be an odd number. Then $\operatorname{disc}\left(\Delta^{d} \mathcal{H}, 3\right) \leq \operatorname{disc}(\mathcal{H}, 3)$ holds for any hypergraph $\mathcal{H}$.

(b) Let $d \geq 2$ be an even number and $c=3 l, l \in \mathbb{N}$. There exists a hypergraph $\mathcal{H}$ with arbitrary large discrepancy that satisfies $\operatorname{disc}\left(\Delta^{d} \mathcal{H}, c\right) \geq \frac{1}{6} \operatorname{disc}(\mathcal{H}, c)^{2}$.

Proof. Obviously $3 \mid k$ ! for all $k \geq 3$. Since $S(d, 2)=2^{d-1}-1$, we have $3 \mid S(d, 2)$ if and only if $d$ is odd. Indeed, $2^{3-1}-1=3,2^{4-1}-1=7$ and if $d=k+2$, then $2^{d-1}-1=4\left(2^{k-1}-1\right)+3$, hence $3 \mid\left(2^{d-1}-1\right)$ if and only if $3 \mid\left(2^{k-1}-1\right)$. Hence Theorem 3 proves both claims. 
Corollary 5. Let $l \in \mathbb{N}$ and $c=4 l$. For all $d \geq 2$ there exists a hypergraph $\mathcal{H}$ with arbitrary large discrepancy such that $\operatorname{disc}\left(\Delta^{d} \mathcal{H}, c\right) \geq \frac{1}{6} \operatorname{disc}(\mathcal{H}, c)^{2}$.

Proof. As $S(d, 2)=2^{d-1}-1$ is an odd number, we have $4 \nmid 2 ! S(d, 2)$. Applying Theorem 3 concludes the proof.

Corollary 6. Let $c \geq 3$ be an odd number and $d \geq 2$. We have

$$
\operatorname{disc}\left(\Delta^{d} \mathcal{H}, c\right) \leq \operatorname{disc}(\mathcal{H}, c) \text { for all hypergraphs } \mathcal{H}
$$

if and only if we have

$$
\operatorname{disc}\left(\Delta^{d} \mathcal{H}, 2 c\right) \leq \operatorname{disc}(\mathcal{H}, 2 c) \text { for all hypergraphs } \mathcal{H} .
$$

Proof. According to Theorem 3, (5) is equivalent to the statement that $c \mid k ! S(d, k)$ for all $k \in\{2, \ldots, d\}$. But, since $2 \mid k$ ! for all $k \geq 2$ and $c$ is odd, this is equivalent to $2 c \mid k ! S(d, k)$ for all $k \in\{2, \ldots, d\}$, which is equivalent to (6).

We now prove the upper bound Theorem 3(i). The main idea is that each hyperedge of the symmetric product intersects all $l$-dimensional simplices with same cardinality. Hence we may color the simplices monochromatically if we can use each color equally often for each $l \geq 2$.

Proof of Theorem 3(i). Let $c, d$ be such that $c \mid k ! S(d, k)$ for all $k \in\{2, \ldots, d\}$. Let $\mathcal{H}=$ $(V, \mathcal{E})$ be a hypergraph and let $\psi: V \rightarrow[c] \operatorname{such}$ that $\operatorname{disc}(\mathcal{H}, \psi)=\operatorname{disc}(\mathcal{H}, c)$. For $X \subseteq V$, put $D(X)=\{(x, \ldots, x) \mid x \in X\}$. We define the following c-coloring $\chi: V^{d} \rightarrow[c]$. For $(v, \ldots, v) \in D(V)$, set $\chi(v, \ldots, v)=\psi(v)$. For the remaining vertices, let $\chi$ be such that all simplices are monochromatic, and for each $k$ there are exactly $\frac{1}{c} k ! S(d, k)$ monochromatic $k$-dimensional simplices in each color.

Let $E \in \mathcal{E}$ and put $R(E):=E^{d} \backslash D(E)$. For any $k \in\{2, \ldots, d\}$ and any two $k$-dimensional simplices $S, S^{\prime}$ we have $|S \cap R(E)|=\left|S^{\prime} \cap R(E)\right|$. Therefore, our choice of $\chi$ implies $\left|\chi^{-1}(i) \cap R(E)\right|=\frac{1}{c}|R(E)|$ for all $i \in[c]$. Hence

$$
\begin{aligned}
& \max _{i \in[c]}|| \chi^{-1}(i) \cap E^{d}\left|-\frac{\left|E^{d}\right|}{c}\right| \\
& =\max _{i \in[c]}|| \chi^{-1}(i) \cap R(E)\left|-\frac{|R(E)|}{c}+\right| \chi^{-1}(i) \cap D(E)\left|-\frac{|D(E)|}{c}\right| \\
& =\max _{i \in[c]}|| \chi^{-1}(i) \cap D(E)\left|-\frac{|D(E)|}{c}\right|=\max _{i \in[c]}|| \psi^{-1}(i) \cap E\left|-\frac{|E|}{c}\right| .
\end{aligned}
$$

This calculation establishes $\operatorname{disc}\left(\Delta^{d} \mathcal{H}, c\right) \leq \operatorname{disc}(\mathcal{H}, c)$. 
To prove the lower bound in Theorem 3, we use the following Ramsey theoretic approach.

Lemma 7. Let $c, d \in \mathbb{N}$. For all $m \in \mathbb{N}$ there exists an $n \in \mathbb{N}$ having the following property: For each c-coloring $\chi:[n]^{d} \rightarrow[c]$ we find a subset $T \subseteq[n]$ with $|T|=m$ such that for all $l \in[d]$ each l-dimensional simplex in $T^{d}$ is monochromatic with respect to $\chi$.

Proof of Lemma 7. The proof is based on an argument from Ramsey theory. First we verify the statement of Lemma 7 for a fixed simplex. Then, by induction over the number of all simplices, we prove the complete assertion of Lemma 7.

Claim: For all $m \in \mathbb{N}$, all $l \in[d]$, all $\sigma \in S_{l}$, and all $J \in P_{l}(d)$, there is an $n \in \mathbb{N}$ such that for all $N \subseteq \mathbb{N}$ with $|N|=n$ and each $c$-coloring $\chi: N^{d} \rightarrow[c]$ there is a subset $T \subseteq N$ with $|T|=m$ and $S_{J}^{\sigma}(T)$ is monochromatic with respect to $\chi$.

Proof of the claim: By Ramsey's theorem (see, e.g. [4], Section 1.2), for every $l \in[d]$ there exists an $n$ such that for each $c$-coloring $\psi:\left(\begin{array}{c}{[n]} \\ l\end{array}\right) \rightarrow[c]$ there is a subset $T$ of $[n]$ with $|T|=$ $m$ and $\left(\begin{array}{l}T \\ l\end{array}\right)$ is monochromatic with respect to $\psi$. Let $N \subseteq \mathbb{N}$ with $|N|=n$. We can assume $N=[n]$ by renaming the elements of $N$ and preserving their order. Let $\chi:[n]^{d} \rightarrow[c]$ be an arbitrary $c$-coloring. We define $\chi_{l, \sigma, J}:\left(\begin{array}{c}{[n]} \\ l\end{array}\right) \rightarrow[c]$ by $\chi_{l, \sigma, J}\left(\left\{x_{1}, \ldots, x_{l}\right\}_{<}\right)=\chi\left(\sum_{i=1}^{l} x_{\sigma(i)} f_{i}\right)$, where the $f_{i}=f_{i}(J)$ are the vectors corresponding to the partition $J$ introduced in Definition 1. By the Ramsey theory argument there is a $T \subseteq N$ with $|T|=m$ and $\chi_{l, \sigma, J}$ is constant on $\left(\begin{array}{l}T \\ l\end{array}\right)$. Hence, $S_{J}^{\sigma}(T)$ is monochromatic with respect to $\chi$. This proves the claim.

Now we derive Lemma 7 from the claim. Each simplex is uniquely determined by a pair

$$
(\sigma, J) \in \bigcup_{l=1}^{d}\left(S_{l} \times P_{l}(d)\right) .
$$

Let $\left(\sigma_{i}, J_{i}\right)_{i \in[s]}$ be an enumeration of all these pairs. Put $n_{0}:=m$. We proceed by induction. Let $i \in[s]$ be such that $n_{i-1}$ is already defined and has the property that for any $N \subseteq \mathbb{N},|N|=n_{i-1}$ and any coloring $\chi: N^{d} \rightarrow[c]$ there is a $T \subseteq N,|T|=m$ such that for all $j \in[i-1], S_{J_{j}}^{\sigma_{j}}(T)$ is monochromatic. Using the claim, we choose $n_{i}$ large enough such that for each $N \subseteq \mathbb{N}$ with $|N|=n_{i}$ and for each $c$-coloring $\varphi: N^{d} \rightarrow[c]$ there exists a subset $T$ of $N$ with $|T|=n_{i-1}$ and $S_{J_{i}}^{\sigma_{i}}(T)$ is monochromatic with respect to $\varphi$. Note that there is a $T^{\prime} \subseteq T,|T|=m$ such that $S_{J_{j}}^{\sigma_{j}}\left(T^{\prime}\right)$ is monochromatic for all $j \in[i]$. Choosing $n:=n_{s}$ proves the lemma.

Related to Lemma 7 is a result of Gravier, Maffray, Renault and Trotignon [5]. They have shown that for any $m \in \mathbb{N}$ there is an $n \in \mathbb{N}$ such that any collection of $n$ different sets contains an induced subsystem on $m$ points such that one of the following holds: (a) each vertex forms a singleton, (b) for each vertex there is a set containing all $m$ points 
except this one, or (c) by sufficiently ordering the points $p_{1}, \ldots, p_{m}$ we have that all sets $\left\{p_{1}, \ldots, p_{\ell}\right\}, \ell \in[m]$, are contained in the system. ${ }^{1}$

In our language, this means that any 0,1 matrix having $n$ distinct rows contains a $m \times$ $m$ submatrix that can be transformed through row and column permutations into a matrix that is (a) a diagonal matrix, (b) the inverse of a diagonal matrix, or (c) a triangular matrix.

Hence this result is very close to the assertion of Lemma 7 for dimension $d=2$ and $c=2$ colors. It is stronger in the sense that not only monochromatic simplices are guaranteed, but also a restriction to 3 of the 8 possible color combinations for the 3 simplices is given. Of course, this stems from the facts that (a) column and row permutations are allowed, (b) not a submatrix with index set $T^{2}$ is provided but only one of type $S \times T$, and (c) the assumption of having different sets ensures sufficiently many entries in both colors.

We are now in the position to prove the second part of Theorem 3.

Proof of Theorem 3(ii). Let $c$ and $d$ be such that $c / k ! S(d, k)$ for some $k \in\{2, \ldots, d\}$. Let $m$ be large enough to satisfy

$$
\frac{1}{2}\left(\begin{array}{c}
m \\
\kappa
\end{array}\right)-\sum_{l=0}^{\kappa-1} l ! S(d, l)\left(\begin{array}{c}
m \\
l
\end{array}\right) \geq \frac{1}{3 k !} m^{k}
$$

for all $\kappa \in\{k, \ldots, d\}$. (This can obviously be done, since the left hand side of the last inequality is of the form $m^{\kappa} / 2 \kappa !+O\left(m^{\kappa-1}\right)$ for $m \rightarrow \infty$.) Using Lemma 7 , we choose $n \in \mathbb{N}$ such that for any $c$-coloring $\chi:[n]^{d} \rightarrow[c]$ there is an $m$-point set $T \subseteq[n]$ with all simplices in $T^{d}$ being monochromatic with respect to $\chi$.

We show that $\mathcal{K}=\left([n],\left(\begin{array}{c}{[n]} \\ m\end{array}\right)\right)$ satisfies our claim. Let $\chi$ be any $c$-coloring of $\mathcal{K}$, choose $T$ as in Lemma 7 . Let $\kappa \in\{k, \ldots, d\}$ be such that for each $l \in\{\kappa+1, \ldots, d\}$ there is the same number of $l$-dimensional simplices in $T$ in each color but not so for the $\kappa$-dimensional simplices. With

$$
\mathcal{S}:=\bigcup_{l=\kappa}^{d} \bigcup_{J \in P_{l}(d)} \bigcup_{\sigma \in S_{l}} S_{J}^{\sigma}(T)
$$

\footnotetext{
${ }^{1}$ To be precise, the authors also have the empty set contained in cases (a) and (c) and the whole set in case (b). It is obvious that by altering $m$ by one, one can transform one result into the other.
} 
we obtain

$$
\begin{aligned}
\operatorname{disc}\left(\Delta^{d} \mathcal{K}, \chi\right) \geq & \max _{i \in[c]}|| \chi^{-1}(i) \cap T^{d}\left|-\frac{\left|T^{d}\right|}{c}\right| \\
\geq & \max _{i \in[c]}\left\{|| \chi^{-1}(i) \cap \mathcal{S}\left|-\frac{|\mathcal{S}|}{c}\right|-|| \chi^{-1}(i) \cap\left(T^{d} \backslash \mathcal{S}\right)\left|-\frac{\left|T^{d} \backslash \mathcal{S}\right|}{c}\right|\right\} \\
\geq & \max _{i \in[c]}\left|\sum_{J \in P_{\kappa}(d), \sigma \in S_{\kappa}}\right| \chi^{-1}(i) \cap S_{J}^{\sigma}(T)\left|-\frac{\kappa ! S(d, \kappa)}{c}\left(\begin{array}{c}
m \\
\kappa
\end{array}\right)\right| \\
& -\frac{c-1}{c}\left(m^{d}-\sum_{l=\kappa}^{d} l ! S(d, l)\left(\begin{array}{c}
m \\
l
\end{array}\right)\right) \\
\geq & \frac{1}{2}\left(\begin{array}{c}
m \\
\kappa
\end{array}\right)-\sum_{l=0}^{\kappa-1} l ! S(d, l)\left(\begin{array}{c}
m \\
l
\end{array}\right) \geq \frac{1}{3 k !} m^{k} .
\end{aligned}
$$

This establishes $\operatorname{disc}\left(\Delta^{d} \mathcal{K}, c\right) \geq \frac{1}{3 k !} m^{k}$. Note that our choice of $n \operatorname{implies} \operatorname{disc}(\mathcal{K}, c)=$ $\left(1-\frac{1}{c}\right) m$.

\section{$3 \quad$ Further Upper Bounds}

Besides the first part of Theorem 3, there are more ways to obtain upper bounds.

Theorem 8. Let $\mathcal{H}=(V, \mathcal{E})$ be a hypergraph. Let $p$ be a prime number, $q \in \mathbb{N}$ and $c=p^{q}$. Furthermore, let $d \geq c$ and $s=d-(p-1) p^{q-1}$. Then $\operatorname{disc}\left(\Delta^{d} \mathcal{H}, c\right) \leq \operatorname{disc}\left(\Delta^{s} \mathcal{H}, c\right)$.

Corollary 9. Let $\mathcal{H}=(V, \mathcal{E})$ be a hypergraph.

(a) If $c$ is a prime number, $q \in \mathbb{N}$ and $d=c^{q}$, then $\operatorname{disc}\left(\Delta^{d} \mathcal{H}, c\right) \leq \operatorname{disc}(\mathcal{H}, c)$.

(b) For arbitrary $d \in \mathbb{N}$ there holds $\operatorname{disc}\left(\Delta^{d} \mathcal{H}, 2\right) \leq \operatorname{disc}(\mathcal{H}, 2)$.

Statement (a) of the corollary follows from the identity $c^{q}=1+(c-1) \sum_{j=0}^{q-1} c^{j}$ and the (repeated) use of Theorem 8. Conclusion (b) follows also from Theorem 8. Note that Theorem 3 implies that in both parts of Corollary 9 we have $c \mid k ! S(d, k)$ for all $k \in\{2, \ldots, d\}$. Hence Corollary 9 could also have been proven by analysing the Stirling numbers.

Proof of Theorem 8. As always, we assume without loss of generality that $V=[n]$. Let us define the shift operator $S:[n]^{d} \rightarrow[n]^{d}$ by

$$
S\left(x_{1}, \ldots, x_{c}, x_{c+1}, \ldots, x_{d}\right)=\left(x_{2}, \ldots, x_{c}, x_{1}, x_{c+1}, \ldots, x_{d}\right) .
$$


It induces an equivalence relation $\sim$ on $[n]^{d}$ by $x \sim y$ if and only if there exists a $k \in[c]$ with $S^{k} x=y$. Now let $x \in[n]^{d}$ and denote its equivalence class by $\langle x\rangle$. Put $k=|\langle x\rangle|$. Obviously $k$ is the minimal integer in $[c]$ with $S^{k} x=x$. A standard argument from elementary group theory ("group acting on a set") shows that $k \mid c$. Thus either $k=c$ or $S^{p^{q-1}} x=x$. Define $D=\left\{y \in[n]^{d}||\langle y\rangle \mid<c\right\}$. Then

$$
\psi: D \rightarrow[n]^{s}, y \mapsto\left(y_{1}, \ldots, y_{p^{q-1}}, y_{c+1}, \ldots, y_{d}\right)
$$

is a bijection. For a given $c$-coloring $\chi$ of $[n]^{s}$, we define a $c$-coloring $\tilde{\chi}$ of $[n]^{d}$ in the following way: We choose a system of representatives $R$ for $\sim$. If $x \in R$ with $|\langle x\rangle|=c$, we put $\tilde{\chi}\left(S^{i} x\right)=i$ for all $i \in[c]$. If $|\langle x\rangle|<c$, then $\tilde{\chi}(y)=(\chi \circ \psi)(y)$ for all $y \in\langle x\rangle$.

Let $E \in \mathcal{E}$. Notice, that $x \in E^{d}$ implies $\langle x\rangle \subseteq E^{d}$, and $x \in D$ implies $\langle x\rangle \subseteq D$. Furthermore, the restriction of $\psi$ to $E^{d} \cap D$ is a bijection onto $E^{s}$. Thus

$$
\begin{aligned}
\max _{i \in[c]}|| \tilde{\chi}^{-1}(i) \cap E^{d}\left|-\frac{\left|E^{d}\right|}{c}\right| \leq & \max _{i \in[c]}|| \tilde{\chi}^{-1}(i) \cap\left(E^{d} \cap D\right)\left|-\frac{\left|E^{d} \cap D\right|}{c}\right| \\
& +\max _{i \in[c]}|| \tilde{\chi}^{-1}(i) \cap\left(E^{d} \backslash D\right)\left|-\frac{\left|E^{d} \backslash D\right|}{c}\right| \\
\leq & \max _{i \in[c]}|| \chi^{-1}(i) \cap E^{s}\left|-\frac{\left|E^{s}\right|}{c}\right|+0 .
\end{aligned}
$$

Hence $\operatorname{disc}\left(\Delta^{d} \mathcal{H}, c\right) \leq \operatorname{disc}\left(\Delta^{s} \mathcal{H}, c\right)$

The following is an extension of the first statement of Theorem 3.

Theorem 10. Let $c, d \in \mathbb{N}$, and let $d^{\prime} \in\{2 \ldots, d\}$. If $c \mid k ! S\left(d^{\prime}, k\right)$ for all $k \in\left\{2, \ldots, d^{\prime}\right\}$, then

$$
\operatorname{disc}\left(\Delta^{d} \mathcal{H}, c\right) \leq \operatorname{disc}\left(\Delta^{d-d^{\prime}+1} \mathcal{H}, c\right)
$$

holds for every hypergraph $\mathcal{H}$.

Proof of Theorem 10. Let $\mathcal{H}=(V, \mathcal{E})$ be a hypergraph with $V=[n]$. Let $\chi:[n]^{d-d^{\prime}+1} \rightarrow$ $[c]$ be an arbitrary $c$-coloring. We define a $c$-coloring $\tilde{\chi}:[n]^{d} \rightarrow[c]$. Let $z \in[n]^{d}, x=$ $\left(z_{1}, \ldots, z_{d^{\prime}}\right)$, and $y=\left(z_{d^{\prime}+1}, \ldots, z_{d}\right)$. If $z_{1}=\ldots=z_{d^{\prime}}=: \zeta$, put $\tilde{\chi}(z)=\chi\left(\zeta, z_{d^{\prime}+1}, \ldots, z_{d}\right)$. Otherwise we find $k \in\left\{2, \ldots, d^{\prime}\right\}, J \in P_{k}\left(d^{\prime}\right)$ and $\sigma \in S_{k}$ with $x \in S_{J}^{\sigma}([n])$. Since $c \mid k ! S\left(d^{\prime}, k\right)$, we can color the set $D:=\left\{\left(z_{\tau(1)}, \ldots, z_{\tau\left(d^{\prime}\right)}, y\right) \mid \tau \in S_{d^{\prime}}\right\}$ of cardinality $k ! S\left(d^{\prime}, k\right)$ evenly by our coloring $\tilde{\chi}:[n]^{d} \rightarrow[c]$. A similar calculation as the one at the end of the proof of Theorem 8 establishes $\operatorname{disc}\left(\Delta^{d} \mathcal{H}, \tilde{\chi}\right) \leq \operatorname{disc}\left(\Delta^{d-d^{\prime}+1} \mathcal{H}, \chi\right)$.

Remark 11. The condition in Theorem 10 is only sufficient but not necessary for the validity of (7), as the following example shows:

Let $c=4, d \geq c$ and $d^{\prime}=3$. According to Theorem 8, we get for each hypergraph $\mathcal{H}$ that $\operatorname{disc}\left(\Delta^{d} \mathcal{H}, c\right) \leq \operatorname{disc}\left(\Delta^{d-2} \mathcal{H}, c\right)=\operatorname{disc}\left(\Delta^{d-d^{\prime}+1} \mathcal{H}, c\right)$. But we have $2 ! S\left(d^{\prime}, 2\right)=6=$ 3 ! $S\left(d^{\prime}, 3\right)$ and $4 \backslash 6$. 
This example shows also, that the methods used in the proofs of Theorem 8 and Theorem 10 are different.

\section{References}

[1] J. Beck and V. T. Sós, Discrepancy theory, in R. Graham, M. Grötschel, and L. Lovász, Editors, Handbook of Combinatorics, Elsevier, Amsterdam, The Netherlands, 1995, 1405-1446.

[2] B. Doerr and A. Srivastav, Multi-Color Discrepancies, Comb. Probab. Comput. 12(2003), 365-399.

[3] B. Doerr, A. Srivastav, and P. Wehr, Discrepancy of Cartesian products of arithmetic progressions, Electron. J. Combin. 11 (2004), Research Paper 5, 16 pp.

[4] R. L. Graham, B. L. Rothschild, and J. H. Spencer, Ramsey Theory, Second Edition, Wiley, New York, USA, 1990.

[5] S. Gravier, F. Maffray, J. Renault, and N. Trotignon, Ramsey-type results on singletons, co-singletons and monotone sequences in large collections of sets, European J. Combin. 25 (2004), 719-734.

[6] J. Riordan, An Introduction to Combinatorial Analysis, Wiley, New York, USA, 1958. 